\title{
(2) OPEN ACCESS \\ Use of saliva flow rate measurement in minor salivary glands autotransplantation for treatment of severe dry eye disease
}

\author{
Jia-Zeng Su ๑ , ${ }^{1}$ Zhen Wang, ${ }^{1,2}$ Xiao-Jing Liu, ${ }^{1}$ Lan Lv, ${ }^{3}$ Guang-Yan Yu ${ }^{4}$
}

\begin{abstract}
'Department of Oral and Maxillofacial Surgery, Peking University School and Hospital of Stomatology, Beijing, China ${ }^{2}$ Department of Stomatology, Capital Medical University Affiliated Beijing Friendship Hospital, Beijing, China ${ }^{3}$ Department of Ophthalmology, Affiliated Beijing Tong Ren Hospital, Capital University of Medical Science, Beijing, China ${ }^{4}$ Department of Oral and Maxillofacial Surgery, Peking University School of Stomatology, Beijing, China
\end{abstract}

\section{Correspondence to} Professor Guang-Yan Yu, Department of Oral and Maxillofacial Surgery, Peking University School of Stomatology, Beijing, China; gyyu@263.netDr Lan Lv; tryklvlan@126.com

J-ZS and ZW contributed equally.

Received 23 July 2020 Revised 21 November 2020 Accepted 23 January 2021
D) Check for updates

(C) Author(s) (or their employer(s)) 2021. Re-use permitted under CC BY-NC. No commercial re-use. See rights and permissions. Published by BMJ.

To cite: Su J-Z, Wang Z, Liu X-J, et al. Br J Ophthalmol Epub ahead of print: [please include Day Month Year]. doi:10.1136/

bjophthalmol-2020-317552

\section{ABSTRACT}

Aims To use minor salivary glands' flow rate (MSGFR) measurement in minor salivary glands (MSGs) autotransplantation for the treatment of severe dry eye disease (DED).

Methods MSGs autotransplantations were performed in 18 eyes ( 17 patients) with severe DED. MSGFR were measured before operation. The upper or lower lip with higher MSGFR was selected as the donor site. Buccal mucosa was the back-up in cases labial MSGs showing markedly decreased MSGFRs. Two pieces of salivary lobules with the covering mucosa were harvested and transplanted to the recipient beds prepared in both upper and lower lids.

Results The donor sites included lower lip in 12 eyes, upper lip in 5 eyes and buccal mucosa in 1 eye. Postoperative follow-up confirmed viable grafts in all cases. The overall subjective relief rate of DED symptoms was $58.8 \%$, with Schirmer test values increasing from 0 $\mathrm{mm}$ to $4 \mathrm{~mm}(\mathrm{p}<0.05)$. The mean preoperative MSGFR was 1.7 (range: $0.9-3.3$ ) $\mu \mathrm{L} / \mathrm{min} / \mathrm{cm}^{2}$. ROC analysis indicated an outstanding discrimination power for preoperative MSGFR to predicate postoperative relief of DED symptoms (area under the curve (AUC) $=0.948$, $\mathrm{p}<0.01)$. The maximum sensitivity $(100 \%)$ and specificity $(72.7 \%)$ were reached at a cut-off of $1.785 \mu \mathrm{L} / \mathrm{min} / \mathrm{cm}^{2}$. Patients with preoperative MSGFR $>1.785 \mu \mathrm{L} / \mathrm{min} / \mathrm{cm}^{2}$ showed greater improvement of Schirmer test values after surgery than those with MSGFR $\leq 1.785 \mu \mathrm{L} / \mathrm{min} / \mathrm{cm}^{2}$ $(p<0.05)$.

Conclusion MSGs transplantation proved to be useful for treating severe DED. The amount of postoperative lubrication and the treatment effect were positively correlated with preoperative MSGFR. MSGFR measurement and donor-site selection should be critical steps before the operation.

\section{INTRODUCTION}

The management of dry eye disease (DED) is a highly complicated process because of its multifactorial aetiology. ${ }^{12}$ Pharmaceutical tear substitutes and topical anti-inflammatory agents are commonly used to treat patients with DED. ${ }^{3}$ Yet, this type of treatment is not adequate for patients with severe DED conditions that are frequently caused by cicatrising conjunctivitis as a result of Stevens-Johnson syndrome, mucous membrane pemphigoid, or chemical burns.

Over the last decade, various methods have been developed to replace the natural lubrication of the eyes. For example, minor salivary glands (MSGs) can be transplanted to the posterior lamella of the eyelids to increase ocular surface lubrication and reduce the discomfort of dry eyes. MSGs transplantation is a relatively simple procedure that can be performed with minimal surgical risks. ${ }^{4}$ The composition of MSG, which is relatively similar to tears, contains large amounts of albumin, immunoglobulins, growth factors, mucins and lipids. ${ }^{4}$ Moreover, the saliva flow rate of MSGs (MSGFR) is 2.1 to $2.9 \mathrm{uL} / \mathrm{min} / \mathrm{cm}^{2}$, which is of the same order of magnitude as the tear flow rate of around 0.6 to $1.4 \mathrm{uL} / \mathrm{min}^{.}{ }^{4-6}$ In addition, the lubrication of saliva from transplanted MSGs used as a tear substitute is spontaneous, continuous and endogenous. Previous studies have shown that the secretions from transplanted MSGs can maintain stable function for more than 4 years (the longest follow-up so far). It can also increase tear production, reduce ocular discomfort and improve tear film stability and other ocular-surface features. ${ }^{4-13}$ Yet, MSGs transplantation is not effective in all patients with DED. Studies have suggested that $73.7 \%$ to $100 \%$ of the patients show Schirmer test improvement, and $53.6 \%$ to $100 \%$ of the patients experience improvement of symptoms after the operation. ${ }^{4-1214}$ The lubrication of the treated eye after MSGs transplantation mainly comes from the saliva secreted by MSGs. The secretory function of MSGs may be severely impaired in some diseases. MSGs transplantation has been widely considered for DED caused by Sjögren's syndrome or StevensJohnson syndrome..$^{7-9} 11$ However, our previous study showed that the MSGFR in patients with Sjögren's syndrome or Stevens-Johnson syndrome was significantly decreased. ${ }^{15}$ Furthermore, our previous data showed that the MSGFR might vary in different subjects and in different oral sites, even for the healthy volunteers. ${ }^{5}$

In this study, we analysed the MSGFR of different sites in patients with DED before MSGs transplantation and accordingly chose the donor sites. The long-term follow-up results and the influence of MSGFR on the treatment effect were also evaluated. Furthermore, we shared our experience in determining the donor size, managing donor wounds, and preventing donor complications.

\section{MATERIALS AND METHODS Patients}

Patients with severe DED were enrolled from June 2010 to October 2014. The inclusion criteria were following: persistently pronounced symptoms 
of dry eye; failure of previous ophthalmological treatments; Schirmer test value of $<2 \mathrm{~mm}$; a break-up time of tear-film (TBUT) value of $<5 \mathrm{~s}$, and positive corneal fluorescein staining (FL) during ophthalmic evaluation. The study was designed and carried out in full accordance with the World Medical Association Declaration of Helsinki (V.2002).

\section{Ophthalmic examinations}

Ophthalmic examinations, including best-corrected visual acuity (BCVA), FL, TBUT and Schirmer test, were performed before surgery. Spectacle correction was applied in BCVA measurement, and the standard logarithmic visual acuity chart was also used. In the FL test, the corneal surface was divided into four quadrants-upper nasal, lower nasal, upper temporal and lower temporal, that were individually scored. The fluorescein score was analysed as follows: $0=$ no staining, $1=$ minimal staining, $2=$ mild $/$ moderate staining and $3=$ severe staining. The sum of the above indicators was taken as the final FL score. In the TBUT test, patients were asked to blink three times after staining. The time from the last time of eye-opening to the appearance of the first dry spot was measured three times, and the mean value was the TBUT score. Schirmer test was performed for 5 min using Whatman No. 41 paper strips without topical anaesthesia.

\section{Patient's questionnaire}

The questionnaire included two items: 'I am satisfied with the long-term treatment effect' and 'My dry eye symptoms were relieved after treatment'. Each item was measured using a 5 -point Likert-type scale that ranged from 'completely disagree' (1 point) to 'completely agree' (5 points). A score of 4 or 5 was regarded as 'subjective relief of DED symptoms' or 'satisfied with the surgery'.

\section{MSGFR measurements}

MSGFR of three sites (upper labial, lower labial and buccal mucosa) was measured as previously described. ${ }^{5}{ }^{15}$ Whatman No. 41 paper strips of $1 \times 2 \mathrm{~cm}$ were used for this procedure. The overlying mucosa of MSGs was carefully dried, and the strip was placed onto the mucosa (figure 1A). Thirty seconds later, the strip was removed and placed in an air-tight container. Both the container and the strip were weighed before and after measurement on an analytical balance (Denver Instrument Co

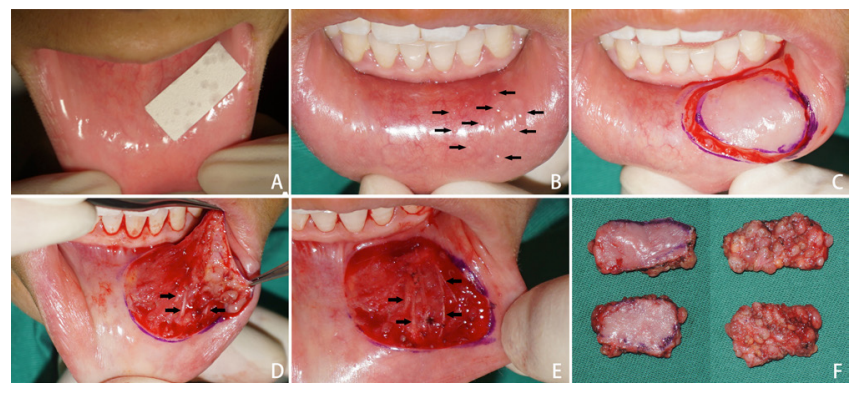

Figure 1 Measurement of the salivary flow rate of minor salivary glands and harvesting of the grafts. (A) Measurement of the salivary flow rate of the donor sites. (B) Observing the donor site to determine the area containing most salivary drops (arrows). (C) Determining the graft's final location according to step B and harvesting of the graft. (D) The incision depth of harvesting the graft (branches of the trigeminal nerve were marked with arrows). (E) The wound of the donor sites (the nerves were marked with arrows). (F) Grafts.

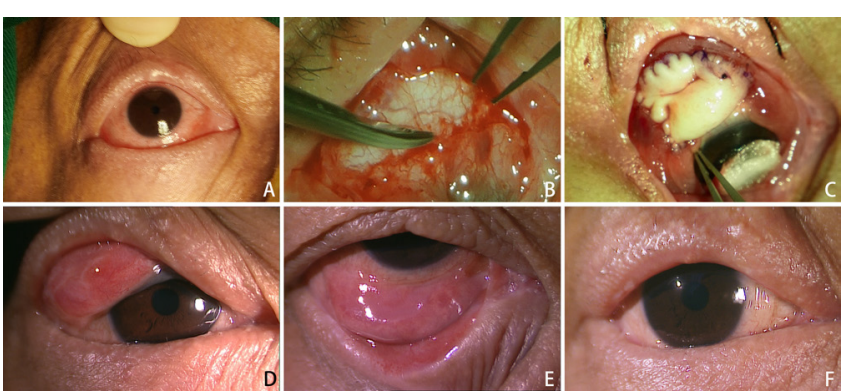

Figure 2 Graft transplantation and patients follow-up. (A) Photo of the dry eye taken before the operation. (B) Preparation of the recipient bed. (C) Fixation of the graft to the recipient bed. ( $D$ and $E$ ) Four years' follow-up of the grafts. (F) Four years' follow-up of the treated eye. The presence of the moisture content on the ocular surface.

Ltd, Beijing). By defining the specific gravity of MSGs saliva as 1, flow rates were calculated and recorded in $\mu \mathrm{L} / \mathrm{min} / \mathrm{cm}^{2}$.

\section{Donors selection}

Considering that the manipulation of harvesting the minor salivary glands in the labial mucosa is more accessible than that in the buccal mucosa, and that protection of the salivary lobules is very important for the donors' postoperative secretory function, the upper or lower labial glands with higher flow rates were selected as the donor sites. In cases where the upper and lower labial MSGFRs were much lower than that of the buccal glands, the latter were selected as donors.

\section{Recipient bed preparation}

All surgeries were performed under general anaesthesia. The recipient beds were prepared and located in both the upper and lower bulbar conjunctiva and near the fornix by the same ophthalmologist, with dissecting scissors (figure 2A,B). No surgical energy instruments or vasoconstrictors were used to protect the microcirculatory flow of the recipient beds. Symblepharon was released, and the incisions extended to the inner and lateral canthus to make the recipient beds as big as possible (figure 2B). Sizes of the recipient beds were measured to determine the sizes of the donors.

\section{Graft harvesting and wound management}

The donor site's mucosa was carefully dried with gauze and exposed under direct vision for 1 to $2 \mathrm{~min}$. The saliva secreted from MSGs was shown as dewdrop (figure 1B). The area containing most salivary drops was chosen as the graft's location (figure 1B,C). According to our experience, the donor tissue size tends to shrink after being totally severed from the donor site. Thus, the graft was finally determined and marked (figure 1C) to a size of about 1.5 times the recipient bed.

The donor was harvested using a scalpel by an oral and maxillofacial surgeon (figure 1C). The incision depth was maintained just at the level between the MSGs lobules and musculus orbicularis oris to prevent injury of the branches of the trigeminal nerve or muscle (figure 1D,E). The graft was finally completely severed from the donor bed and composed of just salivary lobules and the covering mucosa (figure $1 \mathrm{~F}$ ).

The labial wound was repaired with heterogeneous (bovine) acellular dermal matrix, which was approved by the National Medical Products Administration of China in 2007 for human use of soft-tissue defects restoration of the oral cavity (Yantai Zhenghai Bio-tech Co., Ltd.) (15 cases) (figure 3A-D) or local 


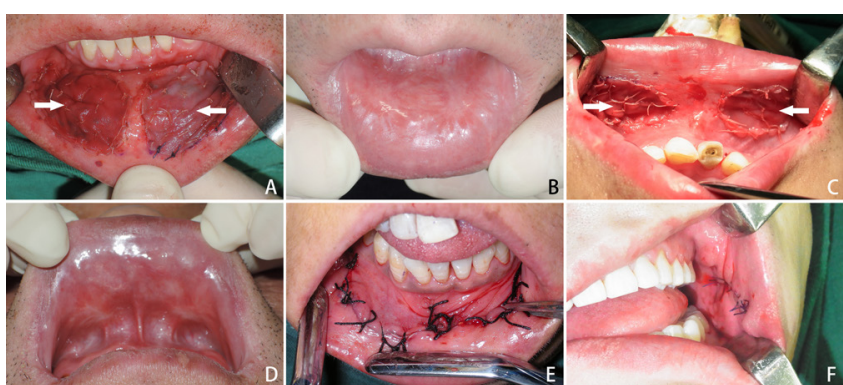

Figure 3 Repair of the donor wound and patients follow-up. (A and B) The repair of the lower lip's wound with acellular dermal matrix (arrows), and 4 years' follow-up. (C and D) The repair of the upper lip's wound with acellular dermal matrix (arrows), and 6.5 years' follow-up. (E) The repair of the wound of the lower lip with local rotational buccal flap. (F) Direct close of the wound of the buccal mucosa.

rotational buccal flap (two cases) (figure 3E). The buccal wound was directly closed (figure $3 \mathrm{~F}$ ).

\section{Graft transplantation}

The covering mucosa of the graft was sutured to the recipient bed with 8-0 absorbable sutures using running suture (figure 2C). The graft was anchored to the underlying orbital septum with one interrupted suture passing through the donor tissue (figure 2C) so as to make good contact between the graft and the underlying recipient bed. No other compression method was used.

\section{Patients follow-up}

Patients were followed-up for 3 months, 1 year and every year after that. Ophthalmic examinations and questionnaires were re-evaluated.

\section{Statistical analysis}

The associations between categorical variables were analysed using the $\chi^{2}$ test. The associations between continuous variables were analysed using the Wilcoxon signed-rank test (two related samples), Mann-Whitney U test (two independent samples) or Kruskal-Wallis test ( $\mathrm{k}$ independent samples). Receiver operating characteristic curve (ROC) analysis was used to evaluate the correlation between preoperative MSGFR and postoperative relief of DED symptoms. All analyses were conducted using SPSS 13.0 (SPSS Inc, Chicago, Illinois, USA). P <0.05 (two-tailed) was considered statistically significant.

\section{RESULTS}

\section{Patients}

MSGs transplantations were performed in 18 eyes (17 patients; 10 women and 7 men); one female patient underwent bilateral transplantations. The aetiology for DED was Stevens-Johnson syndrome in 15 eyes and graft versus host disease in three eyes. The average medical history was 6 years (table 1). In all patients, preoperative conservative measures, including artificial tear substitutes or occlusion of tear drainage, had been proven ineffective in relieving discomfort and visual interference.

\section{Complications}

All eye grafts were successfully transplanted within 2 hours with no recorded intraoperative complication. The mean size of the donor tissues was $8.1 \mathrm{~cm}^{2}$ (IQR: $6.8-10.4 \mathrm{~cm}^{2}$ ). Postoperative follow-up confirmed viable grafts in all cases (figure $2 \mathrm{D}, \mathrm{E}$ ).
Table 1 Baseline and follow-up data of the eyes

\begin{tabular}{|c|c|c|}
\hline & Baseline & $\begin{array}{l}\text { Long-term follow- } \\
\text { up }\end{array}$ \\
\hline \multicolumn{3}{|l|}{ General information } \\
\hline Number of eyes & 18 & - \\
\hline Sex & 11 females, 7 males & - \\
\hline Age & 31 (range: $23-56$ ) years & - \\
\hline Aetiology & 15 SJSs, 3 GVHDs & - \\
\hline Medical history & 6 (range: $0.7-33$ ) years & - \\
\hline \multicolumn{3}{|l|}{ Data of grafts } \\
\hline Donor sites & $\begin{array}{l}12 \text { low lips, } \\
5 \text { upper lips, } \\
1 \text { buccal mucosa }\end{array}$ & - \\
\hline Sizes & 8.1 (range: $5.8-15) \mathrm{cm}^{2}$ & - \\
\hline MSGFR & 1.7 (range: $0.9-3.3$ ) $\mu \mathrm{L} / \mathrm{min} / \mathrm{cm}^{2}$ & - \\
\hline \multicolumn{3}{|l|}{ Patients questionnaire } \\
\hline Follow-up time & - & 6 (range: 4-8) years \\
\hline Number of eyes & - & 18 \\
\hline Relief of DED symptoms & - & $58.8 \%$ * \\
\hline Satisfaction rate & - & $70.6 \%$ * \\
\hline \multicolumn{3}{|l|}{$\begin{array}{l}\text { Ophthalmological } \\
\text { evaluations }\end{array}$} \\
\hline Follow-up time & - & 3.5 (range: 2-8) years \\
\hline Number of eyes & - & $17 \dagger$ \\
\hline Schirmer test & 0 (IQR: 0-0.5) mm & 4 (IQR: 1-7) mm‡ \\
\hline $\mathrm{FL}$ & 12 (IQR: 10-12) & 10 (IQR: 8-12)‡ \\
\hline TBUT & $0 \mathrm{~s}$ & $\begin{array}{l}0 \mathrm{~s} \text { in } 15 \text { eyes, } \\
2 \mathrm{~s} \text { in two eyes }\end{array}$ \\
\hline BCVA & 0.05 (IQR: $0.01-0.1$ ) & 0.05 (IQR: 0.02-0.33) \\
\hline
\end{tabular}

* Only the right eye was included in the patient undergoing bilateral surgeries. tThe ophthalmological examinations of the eye lost to follow-up after 3 months were not included in the analysis. $\ddagger \mathrm{P}<0.05$.

BCVA, best-corrected visual acuity; DED, dry eye disease; FL, corneal fluorescein staining score; GVHD, graft versus host disease; MSGFR, salivary flow rate of minor salivary glands; SJS, Stevens-Johnson syndrome; TBUT, value of break-up time of tear-film.

Partial necrosis of the graft was found 5 days after the operation in one case. The remaining tissue showed good healing after local debridement. The surgical wound of the donor sites healed well in all cases (figure 3B,D). The acellular dermal matrix covering the labial wounds was replaced with autologous tissue. Local transient hypaesthesia of the lower lip was reported in 7 of the 11 cases and persisted for 2 weeks to 6 months (mean: 4 months). No complication was reported in the donor sites of the upper lip or buccal mucosa. A part of the graft in the lower lid was visible after the operation and led to cosmetic problems in two cases. In addition, no cosmetic problems of the lip, superficial gingivolabial sulcus due to scar contraction, or dry mouth were reported.

\section{Patients questionnaire}

Subjective long-term follow-up results (table 1) were obtained for all cases with a mean follow-up time of 6 years (range, 4-8 years). For analysis, only the right eye was included. Subjective relief of DED symptoms (dryness, burning, foreign body sensation, itching and red-eye) occurred in 10 out of the 17 $(58.8 \%)$ eyes. Severe symblepharon was found in seven eyes, and this symptom was relieved in all cases after the operation. The overall subjective satisfaction rate of the surgery was $70.6 \%$ (12/17). Two patients who did not experience noticeable relief 

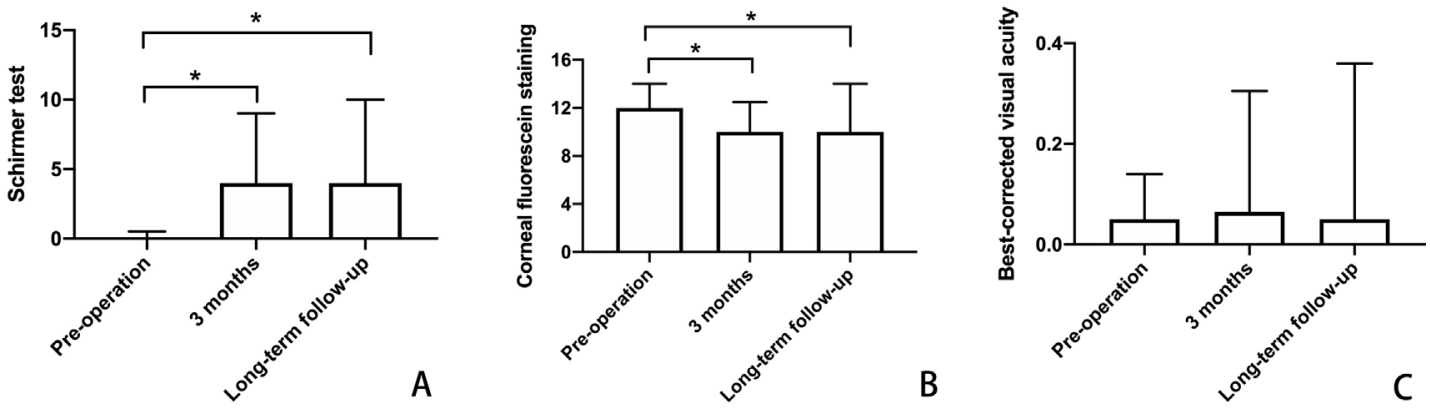

Figure 4 Preoperative and postoperative ophthalmic measurements. (A) Schirmer test values. (B) Fluorescent staining scores. (C) Best-corrected visual acuity. ${ }^{*} \mathrm{P}<0.05$.

from the DED symptoms expressed satisfaction considering they got rid of the symblepharon after the operation.

\section{Ophthalmic evaluations}

Objective follow-up data (table 1 ) of 3 months were obtained for all eyes. A total of $94 \%$ of eyes (17/18) were followed up for more than 2 years (long-term follow-up; data were obtained with a mean duration of 3.5 years) (figure $2 \mathrm{~F}$ ). Schirmer test values significantly increased from a mean preoperative level of $0 \mathrm{~mm}$ (IQR: 0-0.5 mm) to $4 \mathrm{~mm}$ (IQR: $1-6 \mathrm{~mm}$ ) 3 months after surgery and remained virtually unchanged with a mean of $4 \mathrm{~mm}$ (IQR: 1-7 mm) in the long-term follow-up (figure 4A). The mean fluorescent staining scores were significantly reduced from 12 to 10,3 months after surgery, after which they remained stable (figure 4B). TBUT score and BCVA showed no significant differences before and after the operation (figure 4C).

\section{Correlations between grafts and postoperative results}

The donor sites included the lower lip in 12 eyes, the upper lip in 5 eyes and buccal mucosa in 1 eye. The sites showed no influence on either subject questionnaire or ophthalmic examination results (table 2).

The mean preoperative MSGFR for all grafts was 1.7 (range: 0.9-3.3) $\mu \mathrm{L} / \mathrm{min} / \mathrm{cm}^{2}$. ROC analysis was used to evaluate the

\begin{tabular}{|c|c|c|c|c|}
\hline & Lower lip & Upper lip & $\begin{array}{l}\text { Buccal } \\
\text { mucosa }\end{array}$ & $P$ value \\
\hline Number of eyes & 12 & 5 & 1 & \\
\hline \multicolumn{5}{|l|}{3 months follow-up } \\
\hline Schirmer test & $4(1-6) \mathrm{mm}$ & $5(2.5-6.5) \mathrm{mm}$ & $2 \mathrm{~mm}$ & 0.654 \\
\hline $\mathrm{FL}$ & $10(10-12)$ & $10(7-11)$ & 8 & 0.158 \\
\hline TBUT & $0(0-0) \mathrm{s}$ & $0(0-0) \mathrm{s}$ & $0 \mathrm{~s}$ & 0.779 \\
\hline BCVA & $0.1(0.01-0.4)$ & $0.05(0.02-0.6)$ & 0.08 & 0.955 \\
\hline \multicolumn{5}{|l|}{ Long-term follow-up* } \\
\hline Schirmer test & $3(1-5) \mathrm{mm}$ & $7(2.5-7.5) \mathrm{mm}$ & $2 \mathrm{~mm}$ & 0.406 \\
\hline $\mathrm{FL}$ & $10(8-12)$ & $8(8-11)$ & 8 & 0.641 \\
\hline TBUT & $0(0-0) \mathrm{s}$ & $0(0-0) \mathrm{s}$ & $0 \mathrm{~s}$ & 1 \\
\hline BCVA & $0.1(0.01-0.4)$ & $0.05(0.03-0.6)$ & 0.05 & 0.915 \\
\hline $\begin{array}{l}\text { Relief of DED } \\
\text { symptoms }\end{array}$ & $54.5 \% \dagger$ & $80 \%$ & 0 & 0.296 \\
\hline
\end{tabular}

Continuous variables were shown as median (IQR).

*One eye of the lower lip group was lost to follow-up after 3 months. tOnly the right eye was included in the patient undergoing bilateral surgeries. BCVA, best-corrected visual acuity; DED, dry eye disease; FL, corneal fluorescein staining score; TBUT, the value of break-up time of tear-film. correlation between preoperative MSGFR and postoperative relief of DED symptoms (figure 5). The area under the curve (AUC) was 0.948 ( $\mathrm{SE}=0.050,95 \% \mathrm{CI}=0.850$ to 1.000 , $\mathrm{p}=0.002)$, indicating an outstanding discrimination power for preoperative MSGFR to predicate postoperative relief of DED symptoms. The maximum sensitivity (100\%) and specificity $(72.7 \%)$ were reached at a cut-off of $1.785 \mu \mathrm{L} / \mathrm{min} / \mathrm{cm}^{2}$ of preoperative MSGFR.

The eyes were divided into high MSGFR $(>1.785 \mu \mathrm{L} / \mathrm{min} /$ $\left.\mathrm{cm}^{2}\right)$ group $(\mathrm{n}=8)$ and low MSGFR $\left(\leq 1.785 \mu \mathrm{L} / \mathrm{min} / \mathrm{cm}^{2}\right)$ group $(n=10)$. There were no intergroup differences concerning aetiological categories, preoperative ophthalmic examination results or donor sizes et al (table 3). The high MSGFR group had postoperative Schirmer test value of $5 \mathrm{~mm}$ (IQR: 4-6 mm) at 3 months, and $5 \mathrm{~mm}$ (IQR: 4-7 $\mathrm{mm}$ ) at $>2$ years, which were significantly higher than those of the low MSGFR group, with the Schirmer test value of $1 \mathrm{~mm}$ (IQR: $0.5-4 \mathrm{~mm})$ at the 3 -month $(\mathrm{p}=0.012)$ follow-up, and $1 \mathrm{~mm}$ (IQR: $0-5 \mathrm{~mm})$ at the $>2$-year $(\mathrm{p}=0.027)$ follow-up. The postoperative results of FL, TBUT and BCVA showed no significant intergroup differences $(\mathrm{p}>0.05)$ (table 3$)$.

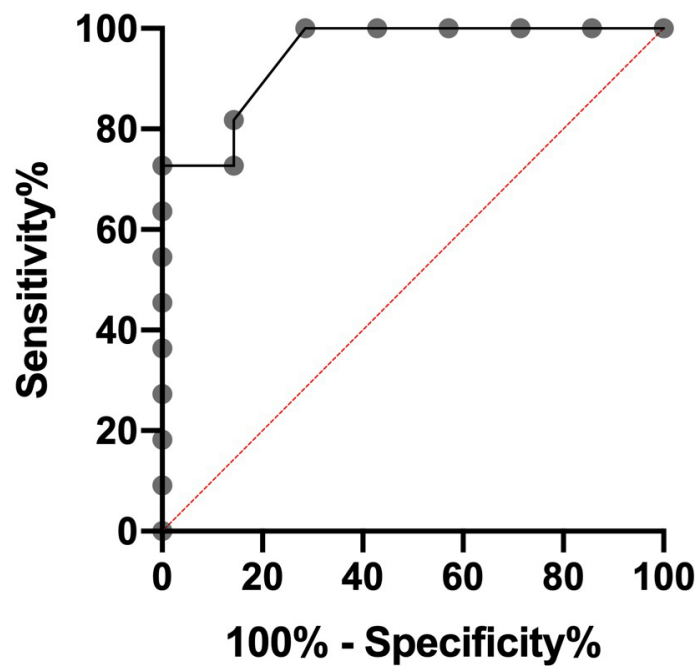

Figure 5 ROC curve of preoperative MSGFR used to predict postoperative relief of DED symptoms. The area under the curve (AUC) was 0.948 ( $\mathrm{SE}=0.050,95 \% \mathrm{Cl}=0.850$ to $1.000, \mathrm{p}=0.002$ ). The optimum cut-off point was defined as the closest point on the ROC curve to the point $(X, Y)=(0,1)$, where $X=1$-specificity and $Y=$ sensitivity, indicating an outstanding discrimination power for preoperative MSGFR to predicate postoperative relief of DED symptoms. The optimum cutoff point of preoperative MSGFR was $1.785 \mu \mathrm{L} / \mathrm{min} / \mathrm{cm}^{2}$. DED, dry eye disease; MSGFR, salivaryflow rate of minor salivary glands. 
Table 3 Comparisons of baseline and follow-up data between low and high MSGFR groups

\begin{tabular}{|c|c|c|c|}
\hline & Low MSGFR group & High MSGFR group & $P$ value \\
\hline \multicolumn{4}{|l|}{ Baseline } \\
\hline Number of eyes & 10 & 8 & \\
\hline Age & $33(24.5-40.5)$ years & $31.5(24-43.75)$ years & 0.897 \\
\hline Sex & 5 males, 5 females & 2 males, 6 females & 0.367 \\
\hline Sides & 6 right eyes, 4 left eyes & 5 right eyes, 3 left eyes & 1.0 \\
\hline Aetiology & 9 SJSs, 1 GVHDs & 6 SJSs, 2 GVHDs & 0.537 \\
\hline Medical history & $15(6-23)$ years & $5(4.25-10.5)$ years & 0.101 \\
\hline Donors sizes & $8(6.49-11.41) \mathrm{cm}^{2}$ & $8.13(6.88-10.18) \mathrm{cm}^{2}$ & 0.829 \\
\hline Schirmer test & $0(0-0) \mathrm{mm}$ & $0(0-2.75) \mathrm{mm}$ & 0.315 \\
\hline $\mathrm{FL}$ & $12(9-12)$ & $12(12-12)$ & 0.315 \\
\hline TBUT & $0(0-0) \mathrm{s}$ & $0(0-0) \mathrm{s}$ & 1.0 \\
\hline BCVA & $0.05(0.01-0.45)$ & $0.09(0.05-0.10)$ & 0.173 \\
\hline \multicolumn{4}{|l|}{3 months follow-up } \\
\hline Number of eyes & 10 & 8 & \\
\hline Schirmer test & $1(0.5-4) \mathrm{mm}$ & $5(4-6) \mathrm{mm}$ & 0.012 \\
\hline $\mathrm{FL}$ & $10(9-12)$ & $10(8.5-10)$ & 0.315 \\
\hline TBUT & $0(0-0) \mathrm{s}$ & $0(0-0) \mathrm{s}$ & 0.696 \\
\hline BCVA & $0.02(0.01-0.44)$ & $0.15(0.06-0.4)$ & 0.068 \\
\hline \multicolumn{4}{|l|}{ Long-term follow-up } \\
\hline Number of eyes & $9^{*}$ & 8 & \\
\hline Follow-up time & $3(3-4.5)$ years & $4.5(2.25-6)$ years & 0.573 \\
\hline Schirmer test & $1(0-5) \mathrm{mm}$ & $5(4-7) \mathrm{mm}$ & 0.027 \\
\hline $\mathrm{FL}$ & $8(8-12)$ & $10(6.5-11.5)$ & 0.673 \\
\hline TBUT & $0(0-0)$ & $0(0-0)$ & 1.0 \\
\hline BCVA & $0.05(0.01-0.43)$ & $0.19(0.1-0.4)$ & 0.139 \\
\hline
\end{tabular}

Continuous variables were shown as median (IQR).

*One eye was lost to follow-up after 3 months.

BCVA, best-corrected visual acuity; DED, dry eye disease; FL, corneal fluorescein staining score; GVHD, graft versus host disease; MSGFR, salivary flow rate of minor salivary glands; SJS, Stevens-Johnson syndrome; TBUT, value of break-up time of tear-film.

\section{DISCUSSION}

In the present study, 18 eyes with severe DED were treated using MSGs transplantation. All the grafts survived, and no severe complications were observed after transplantation. After the operation, the mean values of the Schirmer test improved and remained stable. With the lubrication from the viable grafts, the patient's ocular-surface features improved as validated by FL scores. Also, a subjective relief of the DED symptoms was achieved in $58.8 \%$ cases. The overall subjective satisfaction rate of this surgery was $70.6 \%$. To sum up, these data suggest that MSGs transplantation is a relatively simple surgery and a good treatment choice for some patients with severe refractory DED.

The ROC analysis showed an outstanding discrimination power for preoperative MSGFR to predicate postoperative relief of DED symptoms. Intergroup comparison showed that donors with higher preoperative MSGFR rendered better Schirmer test results after operation. These results indicated that the postoperative lubrication amount and treatment effect was positively correlated with preoperative MSGFR. Besides, our results suggest that measurement of the saliva secretory function of MSGs should be a critical step in selecting patients and donor grafts before the operation. Most of the cases enrolled in the previous studies, as well as in ours, had Stevens-Johnson syndrome, followed by Sjögren syndrome and other local injuries such as chemical burns. ${ }^{4-12}$ However, in patients with Sjögren syndrome, MSGs are involved in lymphocytic infiltration, ${ }^{16}$ and the secretory function is damaged, which results in decreased salivary flow rate. ${ }^{15} 17$ Thus, patients with Sjögren syndrome are not suited for salivary gland transplantation. As for StevensJohnson syndrome, our previous study showed that MSGFR in some patients was also significantly decreased..$^{15}$ Thus, it is crucial to evaluate the secretory function of MSGs in patients with Stevens-Johnson syndrome before transplantation. Sant' Anna et al reported a significant increase in tear production in the eyes that received more than 10 glands per graft compared with those that received fewer glands. ${ }^{11}$ However, if the glands' secretory function is impaired, the graft with big size and more glands might still contribute to poor lubrication. We believe that the MSGFR should be directly measured before transplantation to exclude patients with severely decreased MSGs function.

The ROC analysis provided the sensitivity of $100 \%$ and specificity of $72.7 \%$ for using the cut-off of $1.785 \mu \mathrm{L} / \mathrm{min} / \mathrm{cm}^{2}$ of preoperative MSGFR to predict postoperative relief of DED symptoms. For the same kind of patients with DED of this study, with the similar surgical methods and processes, this cut-off value might be taken as a reference for patients' selection for MSGs transplantation. However, limited by the sample size, we could not conduct multivariate analysis to give more powerful evidence and more universal cut-off of preoperative MSGFR for patients' selection. This is the main limitation of this study.

Graft size was determined according to the most significant capacity of the recipient beds in this study. The mean size was $8.1 \mathrm{~cm}^{2}$, which was much bigger than that reported in other studies. ${ }^{479-11}$ All the grafts survived. For the donor sites, the labial wound was repaired with local rotational buccal flap in the early stage and acellular dermal matrix afterward. Both methods achieved satisfactory results with only mild, temporary complications of the donor sites. In contrast, the acellular dermal matrix would be simpler with lesser damage. Thus, it is safe to harvest and graft a big MSG tissue piece to obtain more lubrication for the eye.

In this study, all surgical operations were performed following the key surgical steps proposed by Geerling et al. ${ }^{4}$ This included protecting the MSG lobules from either mechanical or highenergy injury and the graft's fixation to the recipient bed. Besides, we proposed that the incision depth for harvesting the graft should be just at the level between the MSG lobules and muscles, where the distribution of the sensory nerves was found (see figure 1D,E). This can help to avoid injuries to the muscles and nerves and prevent postoperative cosmetic and sensory complications of the lips, mainly when a big graft is harvested.

Contributors JZS and ZW contributed equally to this work. JZS and GYY obtained funding. JZS, ZW, LL and GYY designed the study. JZS, ZW and XJL collected all the data. JZS, ZW, XJL and LL were involved in data cleaning, mortality follow-up and verification. JZS and ZW analysed the data. JZS drafted the manuscript. JZS, ZW, XIL, LL and GYY contributed to interpreting the results and critical revision of the manuscript for important intellectual content and approved the final version of the manuscript. All authors have read and approved the final manuscript. LL and GYY are the study guarantors and the corresponding authors.

Funding This work was supported by the National Natural Science Foundation of China $(81500873,81974151,81671005)$.

Competing interests None declared.

Patient consent for publication Not required.

Ethics approval The study was approved by the Ethics Committee of the Peking University School of Stomatology (PKUSSIRB - 201412003).

Provenance and peer review Not commissioned; externally peer reviewed.

Data availability statement Data are available upon reasonable request. All data relevant to the study are included in the article or uploaded as supplementary information. All data relevant to the study are included in the article and they are also available from the corresponding author upon reasonable request. 
Open access This is an open access article distributed in accordance with the Creative Commons Attribution Non Commercial (CC BY-NC 4.0) license, which permits others to distribute, remix, adapt, build upon this work non-commercially, and license their derivative works on different terms, provided the original work is properly cited, appropriate credit is given, any changes made indicated, and the use is non-commercial. See: http://creativecommons.org/licenses/by-nc/4.0/.

\section{ORCID iD}

Jia-Zeng Su http://orcid.org/0000-0003-0569-8033

\section{REFERENCES}

1 Labbé A, Wang YX, Jie Y, et al. Dry eye disease, dry eye symptoms and depression: the Beijing eye study. Br J Ophthalmol 2013;97:1399-403.

2 Jones L, Downie LE, Korb D, et al. TFOS DEWS II management and therapy report. Ocul Surf 2017;15:575-628.

3 Sacchetti M, Mantelli F, Lambiase A, et al. Systematic review of randomised clinical trials on topical ciclosporin a for the treatment of dry eye disease. Br J Ophthalmol 2014;98:1016-22.

4 Geerling G, Raus P, Murube J. Minor salivary gland transplantation. Dev Ophthalmol 2008:41:243-54.

5 Wang Z, Shen M-M, Liu X-J, et al. Characteristics of the saliva flow rates of minor salivary glands in healthy people. Arch Oral Biol 2015;60:385-92.

6 Tomlinson A, Khanal S. Assessment of tear film dynamics: quantification approach. Ocul Surf 2005:3:81-95.
7 Murube J, Manyari A, ChenZhuo L. Labial salivary gland transplantation in severe dry eye. Oculoplast Orbital Reconstr Surg 1998;1:104-10.

8 Güerrissi JO, Belmonte J. Surgical treatment of dry eye syndrome: conjunctival graft of the minor salivary gland. J Craniofac Surg 2004;15:6-10.

9 Soares EJC, França VP. [Transplantation of labial salivary glands for severe dry eye treatmentl. Arq Bras Oftalmol 2005;68:481-9.

10 Marinho DR, Burmann TG, Kwitko S. Labial salivary gland transplantation for severe dry eye due to chemical burns and Stevens-Johnson syndrome. Ophthalmic Plast Reconstr Surg 2010;26:182-4.

11 Sant' Anna AEBPP, Hazarbassanov RM, de Freitas D, et al. Minor salivary glands and labial mucous membrane graft in the treatment of severe symblepharon and dry eye in patients with Stevens-Johnson syndrome. Br J Ophthalmol 2012;96:234-9.

12 Wakamatsu TH, Sant'Anna AEBPP, Cristovam PC, et al. Minor salivary gland transplantation for severe dry eyes. Cornea 2017;36 Suppl 1:S26-33.

13 Qin Y, Zhang Y, Liang Q, et al. Labial salivary gland transplantation for severe dry eye in a Rhesus monkey model. Invest Ophthalmol Vis Sci 2018;59:2478-86.

14 Raus P. Radiosurgery AIDS in salivary gland transplants for severe dry eye. Ocular Surg News 2003;12:15-16.

15 Wang Z, Li W, Hong $X$, et al. Minor salivary glands function is decreased in hyposalivation-related diseases. Arch Oral Biol 2016;69:63-70.

16 Kapsogeorgou EK, Christodoulou MI, Panagiotakos DB, et al. Minor salivary gland inflammatory lesions in Sjögren syndrome: do they evolve? J Rheumatol 2013;40:1566-71.

17 Eliasson L, Almståhl A, Lingström P, et al. Minor gland saliva flow rate and proteins in subjects with hyposalivation due to Sjogren's syndrome and radiation therapy. Arch Oral Biol 2005;50:293-9. 